\title{
UNIQUE RANGE SETS FOR POWERS OF MEROMORPHIC FUNCTIONS II
}

\begin{abstract}
S. Mallick, Md T. Anwar. Unique range sets for powers of meromorphic functions II, Mat. Stud. 53 (2020), 134-146.

In this paper, we deal the problems of unique range sets in the light of powers of meromorphic functions and hence answer an open problem posed by Mallick-Sarkar in [15]. We also show the sharpness of our results exhibiting two counter examples. Moreover, our results improve the result of Mallick-Sarkar ([15]).
\end{abstract}

1. Introduction. For two non-constant meromorphic (entire) functions $f, g$ and a complex number $c$ if we have $f^{-1}(c)=g^{-1}(c)$ counting multiplicities, then we say that the functions $f$ and $g$ share the value c counting multiplicities or CM. If we do not count the multiplicity, then we say $f$ and $g$ share the value $c$ ignoring multiplicities or IM. Similar notion for a set also exists in the literature called set sharing ([8]).

For two non-constant meromorphic (entire) functions $f, g$ and a set $S \subseteq \mathbb{C}$ if $f^{-1}(S)=$ $g^{-1}(S)$ counting multiplicities, then we say that the functions $f$ and $g$ share the set $S$ CM. If we do not count the multiplicity, then we say $f$ and $g$ share the set $S$ IM. Henceforth, the notion of unique range set stepped into the literature [9] and in due course of time, it has become one of the most cultivated concepts of the uniqueness theory.

For two arbitrary non-constant meromorphic (entire) functions $f, g$ and a set $S \subseteq \mathbb{C}$ if $f^{-1}(S)=g^{-1}(S)$ CM implies $f \equiv g$, then $S$ is called a unique range set for meromorphic (entire) functions or URSM(URSE) in brief. Further, if $f^{-1}(S)=g^{-1}(S)$ IM implies $f \equiv g$, then $S$ is called a unique range set for meromorphic (entire) functions ignoring multiplicities or URSM-IM(URSE-IM) or RURSM(RURSE) in brief.

Throughout these years, a number of results $[2-7,9]$ concerning URSM(URSE) and URSM-IM(URSE-IM) have been provided by various authors in different time. Consequently, as the time progresses, the prime motto the problem of unique range sets has been confined towards obtaining such sets with smallest possible cardinality. Till date the least possible cardinality for URSM(URSE) [4,6,7] and URSM-IM(URSE-IM) [3,5,7] obtained so far is 11(7) and 17(10) respectively. But in [16, see page 527, Theorem 10.72] and [16, see page 517, Theorem 10.59] we see that the least cardinality of a URSM and URSE must be greater than 5 and 4 respectively. So, for the last two decades it has become an intriguing challenge for the researchers to reduce the existing lower bound of the URSM(URSE) and URSMIM(URSE-IM).

In 2001, Banerjee-Lahiri ([3]) made a further refinement of the notion of unique range sets called URSMk(URSEk), by developing a scaling between the notion of URSM(URSE)

2020 Mathematics Subject Classification: 30D20, 30D30, 30D35.

Keywords: meromorphic function; entire function; uniqueness; unique range set; reduced unique range set. doi:10.30970/ms.53.2.134-146 
and URSM-IM(URSE-IM). This refinement is introduced in line with the notion of weighted sharing $([13,14])$ introduced by Lahiri himself. Below we recall these definitions but for the sake of our convenience we formulate the same in a different style.

Definition 1 ([13,14]). Let $k$ be a nonnegative integer or infinity. For two non-constant meromorphic functions $f, g$ and $a \in \mathbb{C} \cup\{\infty\}$ we denote by $f^{-1}(a, k)$ the set of all a-points of $f$, where an $a$-point of multiplicity $m$ is counted $m$ times if $m \leq k$ and $k+1$ times if $m>k$. If $f^{-1}(a, k)=g^{-1}(a, k)$, we say that $f, g$ share the value a with weight $k$.

We write $f, g$ share $(a, k)$ to mean that $f, g$ share the value $a$ with weight $k$. Clearly if $f, g$ share $(a, k)$, then $f, g$ share $(a, p)$ for any integer $p, 0 \leq p<k$. Also we note that $f, g$ share a value $a \mathrm{IM}$ or $\mathrm{CM}$ if and only if $f, g$ share $(a, 0)$ or $(a, \infty)$ respectively.

Definition 2 ([13,14]). For a non-constant meromorphic function $f$ and $S \subseteq \mathbb{C} \cup\{\infty\}$ we define $f^{-1}(S, k)=\bigcup_{a \in S} f^{-1}(a, k)$, where $k$ is a non-negative integer and $a \in S$.

Then, for two non-constant meromorphic functions $f$ and $g$, by $f^{-1}(S, k)=g^{-1}(S, k)$ we mean that $f$ and $g$ share the set $S$ with weight $k$. Clearly, $f^{-1}(S, \infty)=g^{-1}(S, \infty)$ means $f^{-1}(S)=g^{-1}(S) \mathrm{CM}$ and $f^{-1}(S, 0)=g^{-1}(S, 0)$ means $f^{-1}(S)=g^{-1}(S)$ IM.

Definition 3 ([3]). A set $S \subseteq \mathbb{C} \cup\{\infty\}$ is called a unique range set for meromorphic (entire) functions with weight $k$ if for any two non-constant meromorphic (entire) functions $f$ and $g$, $f^{-1}(S, k)=g^{-1}(S, k)$ implies $f \equiv g$. We write S is $U R S M k(U R S E k)$ in short.

The notion of URSMk renders as a very useful tool to improve the results of URSM(URSE) $([2-4])$. But the improvements were completely based on relaxing the nature of sharing the unique range sets not based on reducing the existing lower bounds of the same. Hence, the least possible cardinality of URSM(URSE) and URSM-IM(URSE-IM) remains unaltered at $11(7)$ and 17(10) respectively. Under this situation, the following question becomes inevitable.

Question 1. Does there at all exist any URSM(URSE) or URSM-IM(URSE-IM) of cardinality less than 11(7) or 17(10) respectively, even for any special class of meromorphic (entire) functions?

Recently, in 2018 Khoai-An-Lai [11], for the first time provided an affirmative answer of Question 1. In fact, they showed that, considering the class of powers of meromorphic functions instead of the class of meromorphic functions, we can even have URSM of cardinality only 7. The result of Khoai-An-Lai [11] is as follows.

Theorem A ([11]). There exist the sets $S$ with 7 elements such that for arbitrary two meromorphic functions $f, g$ and for an integer $d \geq 2$, the condition

$$
\left(f^{d}\right)^{-1}(S, \infty)=\left(g^{d}\right)^{-1}(S, \infty)
$$

implies $f=\xi g$, where $\xi$ is a root of unity of degree $d$.

Later on, in the same year, Mallick-Sarkar ([15]) have generalized and improved the Theorem A by replacing the uniqueness of $f^{d}$ and $g^{d}$ by $f^{d+r}$ and $g^{d+s}$, where $d \in \mathbb{N}$, $r, s \in \mathbb{N} \cup\{0\}$; and by relaxing the nature of sharing the set $S$, respectively. Furthermore in their result, Mallick-Sarkar have also been able to obtain unique range sets of cardinality less than 7 for some special class of meromorphic functions. That is, they have provided a more general and better answer to Question 1 than Theorem B. For that, the authors have introduced the notion of $U R S P^{d} M\left(U R S P^{d} E\right)$ and $U R S P^{d} M k\left(U R S P^{d} E k\right)$ in the literature. So, to proceed further, let us shortly recall these definitions. 
Definition $4([15])$. Suppose $M(\mathbb{C})$ denotes the set of all meromorphic functions defined on $\mathbb{C}$. We define $M^{d}(\mathbb{C})$ to be the collection of all such meromorphic functions which are powers of some meromorphic functions of power at least $d$, where $d$ is a positive integer. That is by set theoretic notation we can write $M^{d}(\mathbb{C})=\left\{f^{d+r} \mid d \in \mathbb{N}, \quad r \in \mathbb{N} \cup\{0\}\right.$ and $\left.f \in M(\mathbb{C})\right\}$.

Clearly, $M^{p}(\mathbb{C}) \subset M^{s}(\mathbb{C}) \subset M^{1}(\mathbb{C})=M(\mathbb{C})$ whenever $p>s>1$.

Similar notions can be defined for entire functions and be denoted by $E(\mathbb{C})$ and $E^{d}(\mathbb{C})$. In that case also we would have $E^{p}(\mathbb{C}) \subset E^{s}(\mathbb{C}) \subset E^{1}(\mathbb{C})=E(\mathbb{C})$ whenever $p>s>1$.

Definition 5 ([15]). Let $f, g \in M^{d}(\mathbb{C})$ be non-constant and $S \subseteq \mathbb{C}$. If $f^{-1}(S)=g^{-1}(S)$ counting multiplicities implies $f \equiv g$, then $S$ is said to be a unique range set for the power of meromorphic(entire) functions with power at least $d$ or $U R S P^{d} M\left(U R S P^{d} E\right)$ in brief.

If we do not take the multiplicities into account then $S$ is said to be a unique range set for the power of meromorphic(entire) functions with power at least $d$ ignoring multiplicities or $U R S P^{d} M$-IM $\left(U R S P^{d} E\right.$-IM) in brief.

Since $M^{p}(\mathbb{C}) \subset M^{s}(\mathbb{C}) \subset M^{1}(\mathbb{C})=M(\mathbb{C})$ whenever $p>s>1$, so we must have $U R S M=U R S P^{1} M$ is a $U R S P^{t} M$ for $t \geq 1$ and every $U R S P^{s} M$ is a $U R S P^{p} M$. Now we define the notion $U R P^{d} M k\left(U R P^{d} E k\right)$ as a gradation between $U R S P^{d} M\left(U R S P^{d} E\right)$ and $U R S P^{d} M-I M\left(U R S P^{d} E-I M\right)$.

Definition 6 ([15]). Let $f, g \in M^{d}(\mathbb{C})$ be non-constant and $S \subseteq \mathbb{C} \cup\{\infty\}$. If $f^{-1}(S, k)=$ $g^{-1}(S, k)$ implies $f \equiv g$, then $S$ is said to be a unique range set with weight $k$ for the power of meromorphic (entire) functions of power at least $d$ or $U R S P^{d} M k\left(U R S P^{d} E k\right)$ in brief.

Thus for $k=\infty$ and $k=0, U R P^{d} M k\left(U R P^{d} E k\right)$ coincides with $U R S P^{d} M\left(U R S P^{d} E\right)$ and $U R S P^{d} M-\mathrm{IM}\left(U R S P^{d} M\right.$-IM $)$ respectively, and for rest of the values of $k$ it runs between these two.

Now we recall the result of Mallick-Sarkar ([15]) as follows.

Theorem B. Let

$$
Q(z)=a z^{n}+b z^{2 m}+c z^{m}+1,
$$

where $n, m \in \mathbb{N}$ and $a, b, c \in \mathbb{C}-\{0\}$ be such that $n>2 m, \operatorname{gcd}(n, m)=1, \frac{c^{2}}{4 b}=\frac{n(n-2 m)}{(n-m)^{2}}$ and $a \neq-\frac{\left(2 m b e_{i}^{2 m}+c m e_{i}^{m}\right)}{n e_{i}^{n}}\left(=\gamma_{i}\right)$ with $e_{i}$ to be the roots of the equation $z^{m}=-\frac{2 n}{(n-m) c}$. Further suppose that $S=\{z: Q(z)=0\}$. Then

(i) $S$ is a $U R S P^{d} M-I M\left(U R S P^{d} E-I M\right)$ for

$$
n>\max \left[m+2+\frac{2}{d}, 2 m+\frac{14}{d}\right]\left(\max \left[m+2,2 m+\frac{7}{d}\right]\right) ;
$$

(ii) $S$ is a $U R S P^{d} M 1\left(U R S P^{d} E 1\right)$ for

$$
n>\max \left[m+2+\frac{2}{d}, 2 m+\frac{9}{d}\right]\left(\max \left[m+2,2 m+\frac{9}{2 d}\right]\right)
$$

(iii) $S$ is a $U R S P^{d} M 2\left(U R S P^{d} E 2\right)$ for

$$
n>\max \left[m+2+\frac{2}{d}, 2 m+\frac{8}{d}\right]\left(\max \left[m+2,2 m+\frac{4}{d}\right]\right) .
$$


In part [ii] of Theorem $\mathrm{B}$, putting $m=1$ and $d \geq 2$, one can easily find that $S$ is a $U R S P^{d} M 1$ for $n \geq 7$, which is a direct improvement of Theorem $\mathrm{A}$ and the notion of $U R S P^{d} M k$ in Theorem B clearly shows the generalization of Theorem A.

Observe that, this new notion of $U R S P^{d} M k\left(U R S P^{d} E k\right)$ provides the existence of URSM (URSE) and URSM-IM(URSE-IM) of cardinality less than 11 and 17 respectively, by allowing the notion of unique range sets for a special class of meromorphic functions. In fact, for some class of powers of meromorphic functions the cardinality of unique range sets can be drastically reduced up to 4 . Though the least cardinality obtained so far for unique range sets in Theorem B is 4 and at the same time the authors could not show the sharpness of their result. So, in the same paper [15], Mallick-Sarkar also posed the following question for the best possible answer to Question 1.

Question 2. How small can we have a set for the uniqueness of two arbitrary functions belonging to any special class of meromorphic functions? If it's cardinality is less than 4, then what is the set?

To answer to Question 2 is the prime objective of the paper. In fact, in this paper, we answer Question 2 affirmatively by providing a $U R S P^{d} M k$ and a $U R S P^{d} E k$ of only three elements, for example $\{-2,3,6\}$. We also exhibit two examples showing the sharpness of our results. That is, the least cardinality of a $U R S P^{d} M k$ or a $U R S P^{d} E k$ can not be less than three.

On the other hand, from Theorem B we see that there exist $U R S P^{d} M 0$, where $d \geq 15$; $U R S P^{d} M 1$, where $d \geq 10 ; U R S P^{d} M 2$, where $d \geq 9 ; U R S P^{d} E 0$, where $d \geq 8$; and $U R S P^{d} E 1$, where $d \geq 5$; of cardinality 4 . But in our main results, we show that there even exist $U R S P^{d} M 0$, where $d \geq 15 ; U R S P^{d} M 1$, where $d \geq 10 ; U R S P^{d} M 2$, where $d \geq 9$; $U R S P^{d} E 0$, where $d \geq 8$; and $U R S P^{d} E 1$, where $d \geq 5$; of cardinality 3 , which is clearly an improvement of Theorem B in the direction of Question 1.

Now we move to the next section for the main results of the paper.

2. Main Results. Let us consider the following polynomial

$$
P(z)=z^{n}+a z^{n-1}+b
$$

where $a$ and $b$ be two non-zero constants such that $P(z)$ has only simple zeros.

Theorem 1. Let $P(z)$ be given by (2), $d(\geq 2)$ be a positive integer and $S=\{z: P(z)=0\}$. Then

(i) $S$ is a $U R S P^{d} M-I M$ for $n>2+\frac{14}{d}$;

(ii) $S$ is a $U R S P^{d} M 1$ for $n>2+\frac{9}{d}$;

(iii) $S$ is a $U R S P^{d} M 2$ for $n>2+\frac{8}{d}$.

Theorem 2. Let $S$ be defined as in Theorem 1 and $d(\geq 1)$ be a positive integer. Then

(i) $S$ is a $U R S P^{d} E-I M$ for $n>2+\frac{7}{d}$;

(ii) $S$ is a $U R S P^{d} E 1$ for $n>2+\frac{9}{2 d}$;

(iii) $S$ is a $U R S P^{d} E 2$ for $n>2+\frac{4}{d}$.

As an application of Theorem 1 and Theorem 2 we can find out uncountably many sets for the uniqueness of the functions $f^{d+r}$ and $g^{d+s}$, where $d \in \mathbb{N}, r, s \in \mathbb{N} \cup\{0\}$ and $f, g$ be two arbitrary non-constant meromorphic functions. Below we provide some of them with least possible cardinality. 
Example 1. Let $S_{5}=\left\{z: z^{5}-3 z^{4}+16=0\right\}$. Now assuming $r=s=0$; for $d=5$ in part-(i), $d=4$ in part-(ii) and $d=3$ in part-(iii) of Theorem 1, we obtain the following examples.

(i) If $f^{5}$ and $g^{5}$ share the set $S_{5}$ ignoring multiplicity, then $f^{5}=g^{5}$.

(ii) If $f^{4}$ and $g^{4}$ share the set $S_{5}$ with weight 1 , then $f^{4}=g^{4}$.

(iii) If $f^{3}$ and $g^{3}$ share the set $S_{5}$ with weight 2 , then $f^{3}=g^{3}$.

Example 2. Let $S_{4}=\left\{z: z^{4}-2 z^{3}+1=0\right\}$. Then for $r=0, s=1$ and $d=8$ in part-(i) and $r=0, s=2$ and $d=5$ in part-(ii) of Theorem 1, we obtain the following examples respectively.

(i) If $f^{8}$ and $g^{9}$ share the set $S_{4}$ ignoring multiplicity, then $f^{8}=g^{9}$.

(ii) If $f^{5}$ and $g^{7}$ share the set $S_{4}$ with weight 1 , then $f^{5}=g^{7}$.

Example 3. Let $S_{3}=\{-2,3,6\}=\left\{z: z^{3}-7 z^{2}+36=0\right\}$. Hence, for $r=s=0$; putting $d=15$ in part-(i), $d=10$ in part-(ii) and $d=9$ in part-(iii) of Theorem 1 , we obtain the following examples.

(i) If $f^{15}$ and $g^{15}$ share the set $S_{3}$ ignoring multiplicity, then $f^{15}=g^{15}$.

(ii) If $f^{10}$ and $g^{10}$ share the set $S_{3}$ with weight 1 , then $f^{10}=g^{10}$.

(iii) If $f^{9}$ and $g^{9}$ share the set $S_{3}$ with weight 2 , then $f^{9}=g^{9}$.

Example 4. For if, $f$ and $g$ are entire functions. Then for any $r, s \in \mathbb{N} \cup\{0\}$ putting $d=3$, $d=4, d=8$ in part (i) and $d=2, d=3, d=5$ in part (ii) of Theorem 2 respectively, we obtain the following examples.

(i) $f^{3+r}$ and $g^{3+s}$ sharing the set $S_{5}$ ignoring multiplicity implies $f^{3+r}=g^{3+s}$.

(ii) $f^{4+r}$ and $g^{4+s}$ sharing the set $S_{4}$ ignoring multiplicity implies $f^{4+r}=g^{4+s}$.

(iii) $f^{8+r}$ and $g^{8+s}$ sharing the set $S_{3}$ ignoring multiplicity implies $f^{8+r}=g^{8+s}$.

(iv) $f^{2+r}$ and $g^{2+s}$ sharing the set $S_{5}$ with weight 1 implies $f^{2+r}=g^{2+s}$.

(v) $f^{3+r}$ and $g^{3+s}$ sharing the set $S_{4}$ with weight 1 implies $f^{3+r}=g^{3+s}$.

(vi) $f^{5+r}$ and $g^{5+s}$ sharing the set $S_{3}$ with weight 1 implies $f^{5+r}=g^{5+s}$.

Remark 1. Note that, all the discussions made above in this section, clearly implies that $\sin ^{5} z$ and $\cos ^{5} z$ or $\sin ^{5} z$ and $\cos ^{6} z$ or $\sin ^{5+r} z$ and $\cos ^{5+s} z$ can never share the set $\{-2,3,6\}$ counting multiplicities and the same will happen for 5 th or more power of any two distinct entire functions.

Similar conclusions can be made for different sharing notions and sets discussed above.

Now we provide the following two examples showing that there can not exist a singleton set or a set with two elements for the uniqueness of two powers of meromorphic functions. That is, Theorem 1 and Theorem 2 are sharp.

Example 5. Suppose that $S=\{a\}$, where $a \neq 0$. Consider $f=e^{z}$ and $g=a^{\frac{2}{d}} e^{-z}$, where $d \in \mathbb{N}$ and by $a^{\frac{2}{d}}$ we mean exactly one of the values of the $d$ th roots of $a^{2}$. Then clearly $f^{d}$ and $g^{d}$ share $S$ CM but $f^{d} \neq g^{d}$.

If $a=0$, then we see that for $f=e^{z}$ and $g=e^{-z} ; f^{d}$ and $g^{d}$ share $S$ CM but $f^{d} \neq g^{d}$. 
Example 6. Suppose that $S=\{\alpha, \beta\}$, where $\alpha, \beta$ both are non-zero complex numbers. Consider $f=e^{z}$ and $g=(\alpha \beta)^{\frac{1}{d}} e^{-z}$, where $d \in \mathbb{N}$ and by $(\alpha \beta)^{\frac{1}{d}}$ we mean exactly one of the values of the $d$ th roots of $\alpha \beta$. Then clearly $f^{d}$ and $g^{d}$ share $S$ CM but $f^{d} \neq g^{d}$.

If one of $\alpha, \beta$ is zero, say $\alpha=0$, then for $f=(\beta)^{\frac{1}{d}} e^{z}, g=(\beta)^{\frac{1}{d}} e^{-z}$; we get that $f^{d}$ and $g^{d}$ share $S$ CM but $f^{d} \neq g^{d}$.

Remark 2. From Theorem 1 and Theorem 2 one can easily find that there exist $U R S P^{d} M 0$, where $d \geq 15 ; U R S P^{d} M 1$, where $d \geq 10 ; U R S P^{d} M 2$, where $d \geq 9 ; U R S P^{d} E 0$, where $d \geq 8$; and $U R S P^{d} E 1$, where $d \geq 5$; of cardinality 3 . But according to Theorem $\mathrm{B}$ we can have these unique range sets of cardinality at least 4. Not only that, comparing Theorem 1 and Theorem 2 with Theorem B one would easily find that for a value of $d$; Theorem 1 and Theorem 2 always provide same or better estimations of $n$ than Theorem B. Hence, clearly Theorem 1 and Theorem 2 together improve Theorem B in the direction of Question 1 as well as answer Question 2.

3. Lemmas. In this section, we present different lemmas which are required to prove the main results of the paper. Before that, we recall the following definitions of different notations which we use in different lemmas and in the proofs of the main theorems. For standard notations and definitions of Nevanlinna Theory we refer our readers to follow $[10,16]$.

Definition 7 ([12]). For $a \in \mathbb{C} \cup\{\infty\}$ we denote by $N(r, a ; f \mid=1)$ the counting function of simple $a$-points of $f$. For a positive integer $m$ we denote by $N(r, a ; f \mid \leq m)(N(r, a ; f \mid \geq m))$ the counting function of those $a$-points of $f$ whose multiplicities are not greater (less) than $m$, where each $a$-point is counted according to its multiplicity.

$\bar{N}(r, a ; f \mid \leq m)(\bar{N}(r, a ; f \mid \geq m))$ are defined similarly, where in counting the $a$-points of $f$ we ignore the multiplicities.

Also, $N(r, a ; f \mid<m), N(r, a ; f \mid>m), \bar{N}(r, a ; f \mid<m)$ and $\bar{N}(r, a ; f \mid>m)$ are defined analogously.

Definition 8 ([18]). Let $f$ and $g$ be two non-constant meromorphic functions such that $f$ and $g$ share $(a, 0)$, where $a \in \mathbb{C} \cup\{\infty\}$. Let $z_{0}$ be an $a$-point of $f$ with multiplicity $p$, an $a$-point of $g$ with multiplicity $q$. We denote by $\bar{N}_{L}(r, a ; f)\left(\bar{N}_{L}(r, a ; g)\right)$ the reduced counting function of those $a$-points of $f$ and $g$ where $p>q(q>p)$, by $N_{E}^{1)}(r, a ; f)\left(N_{E}^{1)}(r, a ; g)\right)$ the counting function of those $a$-points of $f$ and $g$ where $p=q=1$. Clearly when $f$ and $g$ share $(a, m), m \geq 1$, then $N_{E}^{1)}(r, a ; f)=N(r, a ; f \mid=1)$.

Definition $9([13,14])$. Let $f, g$ share $(a, 0)$. We denote by $\bar{N}_{*}(r, a ; f, g)$ the reduced counting function of those $a$-points of $f$ whose multiplicities differ from the multiplicities of the corresponding $a$-points of $g$.

Clearly $\bar{N}_{*}(r, a ; f, g)=\bar{N}_{*}(r, a ; g, f)=\bar{N}_{L}(r, a ; f)+\bar{N}_{L}(r, a ; g)$.

Suppose

$$
\begin{array}{cl}
f_{1}^{d+r}=f, & g_{1}^{d+s}=g \\
F=\frac{f^{n-1}(f+a)}{-b}, & G=\frac{g^{n-1}(g+a)}{-b},
\end{array}
$$

where $f_{1}, g_{1}$ be two arbitrary non-constant meromorphic functions with $d \in \mathbb{N}$ and $r, s \in$ $\mathbb{N} \cup\{0\}$. Clearly $f, g \in M^{d}(\mathbb{C})$. Henceforth, we shall denote by $H$ the following function.

$$
H=\left(\frac{F^{\prime \prime}}{F^{\prime}}-\frac{2 F^{\prime}}{F-1}\right)-\left(\frac{G^{\prime \prime}}{G^{\prime}}-\frac{2 G^{\prime}}{G-1}\right) .
$$


Lemma 1 ([19]). If $F, G$ are two non-constant meromorphic functions such that they share $(1,0)$ and $H \not \equiv 0$, then

$$
N_{E}^{1)}(r, 1 ; F) \leq N(r, H)+S(r, F)+S(r, G) .
$$

Lemma 2 ([1]). Let $f$ and $g$ be two meromorphic functions sharing $(1, l)$, where $0 \leq l<\infty$. Then

$$
N(r, 1 ; f)+\bar{N}(r, 1 ; g)-N_{E}^{1)}(r, 1 ; f)+\left(l-\frac{1}{2}\right) \bar{N}_{*}(r, 1 ; f, g) \leq \frac{1}{2}[N(r, 1 ; f)+N(r, 1 ; g)] .
$$

Lemma 3. Let $F$ and $G$ be given by (4) and $H \not \equiv 0$. If $F, G$ share $(1,0)$, then

$$
\begin{gathered}
N(r, H) \leq \bar{N}(r, 0 ; f)+\bar{N}\left(r,-a \frac{(n-1)}{n} ; f\right)+\bar{N}(r, \infty ; f) \\
+\bar{N}(r, 0 ; g)+\bar{N}\left(r,-a \frac{(n-1)}{n} ; g\right)+\bar{N}(r, \infty ; g)+ \\
+\bar{N}_{*}(r, 1 ; F, G)+\bar{N}_{0}\left(r, 0 ; f^{\prime}\right)+\bar{N}_{0}\left(r, 0 ; g^{\prime}\right)+S(r, f)+S(r, g),
\end{gathered}
$$

where $\bar{N}_{0}\left(r, 0 ; f^{\prime}\right)$ denotes the reduced counting function corresponding to the zeros of $f^{\prime}$ which are not the zeros of $f\left(f+a \frac{(n-1)}{n}\right)(F-1) . \bar{N}_{0}\left(r, 0 ; g^{\prime}\right)$ is defined similarly.

Proof. Since $F, G$ share $(1,0)$ and $H$ has only simple poles, therefore the result is obvious by some simple calculations. We omit the details.

Lemma 4. Let $F$ and $G$ be given by (4) and $H \not \equiv 0$. If $F, G$ share $(1, l)$, then

$$
\begin{gathered}
\left(\frac{n}{2}-1\right)[T(r, f)+T(r, g)] \leq 2[\bar{N}(r, 0 ; f)+\bar{N}(r, 0 ; g)]+ \\
+2[\bar{N}(r, \infty ; f)+\bar{N}(r, \infty ; g)]-\left(l-\frac{3}{2}\right) \bar{N}_{*}(r, 1 ; F, G)+S(r, f)+S(r, g) .
\end{gathered}
$$

Proof. By the Second Fundamental Theorem we get

$$
\begin{aligned}
(n+1) T(r, f) \leq \bar{N}(r, 1 ; F) & +\bar{N}(r, \infty ; f)+\bar{N}(r, 0 ; f)+\bar{N}\left(r,-a \frac{(n-1)}{n} ; f\right)- \\
& -N_{0}\left(r, 0 ; f^{\prime}\right)+S(r, f), \\
(n+1) T(r, g) \leq \bar{N}(r, 1 ; G)+ & \bar{N}(r, \infty ; g)+\bar{N}(r, 0 ; g)+\bar{N}\left(r,-a \frac{(n-1)}{n} ; g\right)- \\
& -N_{0}\left(r, 0 ; g^{\prime}\right)+S(r, g) .
\end{aligned}
$$

Now combining (5), (6) and using Lemma 2, Lemma 1 and Lemma 3 we obtain

$$
\begin{aligned}
(n+1) & {[T(r, f)+T(r, g)] \leq \bar{N}(r, 1 ; F)+\bar{N}(r, 1 ; G)+\bar{N}(r, \infty ; f)+\bar{N}(r, \infty ; g)+} \\
& +\bar{N}(r, 0 ; f)+\bar{N}(r, 0 ; g)+\bar{N}\left(r,-a \frac{(n-1)}{n} ; f\right)+\bar{N}\left(r,-a \frac{(n-1)}{n} ; g\right)- \\
& -N_{0}\left(r, 0 ; g^{\prime}\right)-N_{0}\left(r, 0 ; f^{\prime}\right)+S(r, f)+S(r, g) \leq \frac{n}{2}[T(r, f)+T(r, g)]+ \\
+ & {[\bar{N}(r, 0 ; f)+\bar{N}(r, 0 ; g)]+2\left[\bar{N}\left(r,-a \frac{(n-1)}{n} ; f\right)+\bar{N}\left(r,-a \frac{(n-1)}{n} ; g\right)\right]-} \\
& -\left(l-\frac{3}{2}\right) \bar{N}_{*}(r, 1 ; F, G)+2[\bar{N}(r, \infty ; f)+\bar{N}(r, \infty ; g)]+S(r, f)+S(r, g),
\end{aligned}
$$

which proves the lemma. 
Lemma 5. Let $F$ and $G$ be given by (4). If $F, G$ share $(1, l)$, where $0 \leq l<\infty$. Then $\bar{N}_{*}(r, 1 ; F, G) \leq \frac{1}{l+1}[\bar{N}(r, 0 ; f)+\bar{N}(r, \infty ; f)+\bar{N}(r, 0 ; g)+\bar{N}(r, \infty ; g)]+S(r, f)+S(r, g)$.

Proof. Using $\bar{N}_{*}(r, 1 ; F, G)=\bar{N}_{L}(r, 1 ; F)+\bar{N}_{L}(r, 1 ; G)$, the lemma can be proved same as in the line of the proof of Lemma 2.12 in [1].

Lemma 6. Let $F$ and $G$ be defined by (4). Then $F G \neq 1$ for $n>4$, when $d \leq 2$ and $n>1+\frac{2}{d-2}$, when $d>2$.

Proof. If possible suppose that $F G=1$. That is

$$
f^{n-1}(f+a) g^{n-1}(g+a)=b^{2} .
$$

Clearly applying the first fundamental theorem on (7) we would get

$$
T(r, f)=T(r, g)+O(1) .
$$

Now we pass through the following subcases to complete the proof of this lemma.

Case-1. Suppose $d \leq 2$. Clearly from (7) each pole of $f$ is a zero of $g$ or $-a$ point of $g$ and vice-versa. Suppose $z_{0}$ is a pole of $f$ of order $p$ and zero of $g$ of order $q$. Then $n p=(n-1) q$, which clearly implies $q>p$. Hence $q=n(q-p) \geq n$. Therefore, $\bar{N}(r, 0 ; g) \leq \frac{1}{n} N(r, 0 ; g)$. Applying similar arguments to the $-a$ points of $g$ we would have $\bar{N}(r,-a ; g) \leq \frac{1}{n} N(r,-a ; g)$. Now for 0 and $-a$ point of $f$ proceeding in the same way as done above for 0 and $-a$ point of $g$, we obtain $\bar{N}(r, 0 ; f) \leq \frac{1}{n} N(r, 0 ; f)$ and $\bar{N}(r,-a ; f) \leq \frac{1}{n} N(r,-a ; f)$. Therefore, using the Second Fundamental Theorem in view of $(8)$ we get

$$
T(r, f) \leq \bar{N}(r, 0 ; f)+\bar{N}(r, \infty ; f)+\bar{N}(r,-a ; f)+S(r, f) \leq \frac{4}{n} T(r, f)+S(r, f),
$$

which is a contradiction for $n>4$.

Case-2. Suppose $d>2$. Then from (3) we get $\bar{N}(r, 0 ; f) \leq \frac{1}{d} N(r, 0 ; f)$ and $\bar{N}(r, \infty ; f) \leq$ $\frac{1}{d} N(r, \infty ; f)$. Also we obtain $\bar{N}(r,-a ; f) \leq \frac{1}{n} N(r,-a ; f)$ like Case- 1 . Then using the Second Fundamental Theorem we get

$$
T(r, f) \leq \bar{N}(r, 0 ; f)+\bar{N}(r, \infty ; f)+\bar{N}(r,-a ; f)+S(r, f) \leq\left(\frac{2}{d}+\frac{1}{n}\right) T(r, f)+S(r, f),
$$

which is a contradiction for $n>1+\frac{2}{d-2}$.

Remark 3. Observe that in the above lemma, if we consider $f$ and $g$ to be entire functions, then $f$ would not have any pole. Hence from (7) we have $g$ omits $0,-a$, which is a contradiction for $n \geq 2$ as $g$ is non-constant. Therefore, $F G \neq 1$ for $n \geq 2$ and for all $d \in \mathbb{N}$.

Lemma 7. Let $F$ and $G$ be defined by (4). Then $F \equiv G$ implies $f \equiv g$ for $n>\frac{3}{2}+\frac{2}{d-1}$, where $d>1$.

Proof. Since $F \equiv G$. Therefore we have

$$
f^{n-1}(f+a)=g^{n-1}(g+a)
$$


By substituting $h=\frac{g}{f}$ in the above equation we get

$$
f^{n}\left(1-h^{n}\right)+a f^{n-1}\left(1-h^{n-1}\right)=0 .
$$

If $h$ is non-constant, then from (10) we have

$$
f=-a \frac{h^{n-1}-1}{h^{n}-1}=-a \frac{\prod_{i=1}^{n-2}\left(h-\alpha_{i}\right)}{\prod_{i=1}^{n-1}\left(h-\beta_{i}\right)}
$$

where $\alpha_{i}$ 's are distinct $(n-1)$ th roots of unity with $\alpha_{i} \neq 1$ and $\beta_{i}$ 's are distinct $n$th roots of unity with $\beta_{i} \neq 1$. Clearly $\alpha_{i} \neq \beta_{j}$. Since $f=f_{1}^{d+r}$, so each $\alpha_{i}$ and $\beta_{i}$ point of $h$ is of multiplicity at least $d$. Further, we have

$$
h=\frac{f}{g}=\frac{f_{1}^{d+r}}{g_{1}^{d+s}} .
$$

Since (9) implies that $f$ and $g$ share $\infty$ CM, so zeros of $h$ come from the zeros of $f$. From (9) we also get that zeros of $f$ come from zeros of $g$ or $-a$ points of $g$. Clearly one can easily verify that those zeros of $f$ which are zeros of $g$ must be of same multiplicity. Hence none of such zeros of $f$ can contribute to the zeros of $h$. Therefore zeros of $h$ are precisely those zeros of $f$ which are the $-a$ points of $g$ and in view of (12) those zeros of $h$ are of multiplicity at least $d$. Repeating similar arguments to the poles of $h$ we shall obtain that each pole of $h$ is of multiplicity at least $d$. Hence using the Second Fundamental Theorem we get

$$
\begin{gathered}
(2 n-3) T(r, h) \leq \sum_{i=1}^{n-2} \bar{N}\left(r, \alpha_{i} ; h\right)+\sum_{i=1}^{n-1} \bar{N}\left(r, \beta_{i} ; h\right)+\bar{N}(r, 0 ; h)+\bar{N}(r, \infty ; h)+S(r, h) \leq \\
\leq \frac{1}{d} \sum_{i=1}^{n-2} N\left(r, \alpha_{i} ; h\right)+\frac{1}{d} \sum_{i=1}^{n-1} N\left(r, \beta_{i} ; h\right)+\frac{1}{d} N(r, 0 ; h)+\frac{1}{d} N(r, \infty ; h)+S(r, h) \leq \\
\leq \frac{(2 n-1)}{d} T(r, h)+S(r, h),
\end{gathered}
$$

which is a contradiction for $n>\frac{3}{2}+\frac{2}{d-1}$. Thus $h$ is a constant, which implies $h^{n}=h^{n-1}=1$; i.e., $h=1$ and hence $f \equiv g$.

Remark 4. Note that if $f$ and $g$ are entire functions in Lemma 7, then from (11) one can easily conclude that $h$ omits $\beta_{i}$ points for $i=1,2, \ldots, n-1$, where $n \geq 4$, which contradicts the fact that $h$ is non-constant. Hence we would have $f \equiv g$ for $n \geq 4$ even if $d=1$. For $d>1$ we may also consider the above result for entire functions.

\section{Proof of the theorems.}

Proof of Theorem 1. [i] Let $F$ and $G$ be defined by (4). Then $F, G$ share $(1,0)$.

Case-1. Suppose $H \not \equiv 0$. Then using Lemma 5 for $l=0$ in Lemma 4 we get

$$
\left(\frac{n}{2}-1\right)[T(r, f)+T(r, g)] \leq \frac{3}{2} \bar{N}_{*}(r, 1 ; F, G)+2[\bar{N}(r, 0 ; f)+\bar{N}(r, 0 ; g)]+
$$




$$
\begin{gathered}
+2[\bar{N}(r, \infty ; f)+\bar{N}(r, \infty ; g)]+S(r, f)+S(r, g) \leq \\
\leq\left(\frac{3}{2}\right)[\bar{N}(r, 0 ; f)+\bar{N}(r, 0 ; g)+\bar{N}(r, \infty ; f)+\bar{N}(r, \infty ; g)]+ \\
+\frac{4}{d}[T(r, f)+T(r, g)]+S(r, f)+S(r, g) \leq\left(\frac{7}{d}\right)[T(r, f)+T(r, g)]+S(r, f)+S(r, g),
\end{gathered}
$$

which is a contradiction for $n>2+\frac{14}{d}$.

Case-2. Suppose $H \equiv 0$. Then on integration we get

$$
\frac{1}{F-1}=\frac{A}{G-1}+B
$$

where $A(\neq 0), B$ are complex constants. From (13), clearly we have

$$
T(r, f)=T(r, g)+S(r, g) .
$$

Now we can write (13) as

$$
F=\frac{(B+1) G+A-B-1}{B G+A-B} .
$$

Hence let us consider the following subcases.

Subcase-2.1. Let $B \neq 0$.

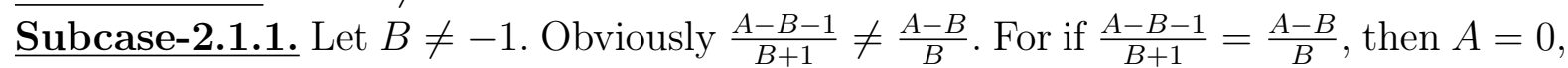
which is absurd. Therefore

$$
\bar{N}\left(r, \frac{B-A}{B} ; G\right)=\bar{N}(r, \infty ; F)
$$

Now using the Second Fundamental Theorem we have

$$
\begin{gathered}
T(r, G) \leq \bar{N}(r, 0 ; G)+\bar{N}(r, \infty ; G)+\bar{N}\left(r, \frac{B-A}{B} ; G\right)+S(r, G) \leq \\
\leq \bar{N}(r, 0 ; g)+\bar{N}(r,-a ; g)+\bar{N}(r, \infty ; g)+\bar{N}(r, \infty ; f)+S(r, G) \leq\left(\frac{1+\frac{3}{d}}{n}\right) T(r, G)+S(r, G),
\end{gathered}
$$

which is a contradiction for $n>1+\frac{3}{d}$.

Subcase-2.1.2. Let $B=-1$. Then from (15) we get

$$
F=\frac{A}{-G+A+1} \text {. }
$$

Subcase-2.1.2.1. Let $A+1 \neq 0$. Then $\bar{N}(r, A+1 ; G)=\bar{N}(r, \infty ; F)$ and of course $\bar{N}(r, \infty ; G)=\bar{N}(r, 0 ; F)$. Now using the Second Fundamental Theorem we have

$$
\begin{aligned}
T(r, G) & \leq \bar{N}(r, 0 ; G)+\bar{N}(r, \infty ; G)+\bar{N}(r, A+1 ; G)+S(r, G) \\
& \leq \bar{N}(r, 0 ; g)+\bar{N}(r,-a ; g)+\bar{N}(r, \infty ; g)+\bar{N}(r, \infty ; f)+S(r, G) \\
& \leq\left(\frac{1+\frac{3}{d}}{n}\right) T(r, G)+S(r, G),
\end{aligned}
$$


which is a contradiction for $n>1+\frac{3}{d}$.

Subcase-2.1.2.2. Let $A+1=0$. Then $F G=1$. Since $n>2+\frac{14}{d}$, so in view of Lemma 6 , this case is invalid.

Subcase-2.2. Suppose $B=0$ then from (15) we get

$$
A F=G+A-1
$$

Subcase-2.2.1. Let $A \neq 1$. Therefore (18) implies $\bar{N}(r, 0 ; F)=\bar{N}(r, 1-A ; G)$. Now using the Second Fundamental Theorem in view of (14) we get

$$
\begin{gathered}
T(r, G) \leq \bar{N}(r, 0 ; G)+\bar{N}(r, \infty ; G)+\bar{N}(r, 1-A ; G)+S(r, G) \leq \\
\leq \bar{N}(r, 0 ; g)+\bar{N}(r,-a ; g)+\bar{N}(r, \infty ; g)+\bar{N}(r, 0 ; f)+\bar{N}(r,-a ; f)+S(r, G) \leq \\
\leq\left(\frac{2+\frac{3}{d}}{n}\right) T(r, G)+S(r, G),
\end{gathered}
$$

which is a contradiction for $n>2+\frac{3}{d}$.

Subcase-2.2.2. Let $A=1$ i.e., $F \equiv G$. So in view of Lemma 7 , we get $f \equiv g$; i.e., $f_{1}^{d+r}=g_{1}^{d+s}$ as $n>2+\frac{14}{d}$.

[ii] Let $F$ and $G$ be defined by (4). Then $F, G$ share $(1,1)$.

Case-1. Suppose $H \not \equiv 0$. Then using Lemma 5 for $l=1$ in Lemma 4 we get

$$
\begin{gathered}
\left(\frac{n}{2}-1\right)[T(r, f)+T(r, g)] \leq \frac{1}{2} \bar{N}_{*}(r, 1 ; F, G)+2[\bar{N}(r, 0 ; f)+\bar{N}(r, 0 ; g)]+ \\
+2[\bar{N}(r, \infty ; f)+\bar{N}(r, \infty ; g)]+S(r, f)+S(r, g) \leq \\
\leq\left(\frac{1}{2}\right)\left(\frac{1}{2}\right)[\bar{N}(r, 0 ; f)+\bar{N}(r, 0 ; g)+\bar{N}(r, \infty ; f)+\bar{N}(r, \infty ; g)]+ \\
+\frac{4}{d}[T(r, f)+T(r, g)]+S(r, f)+S(r, g) \leq\left(\frac{9}{2 d}\right)[T(r, f)+T(r, g)]+S(r, f)+S(r, g),
\end{gathered}
$$

which is a contradiction for $n>2+\frac{9}{d}$.

Case-2. Suppose $H \equiv 0$. Then this case can be dealt same as in the line of proof of Case-2 of Part [i] of this theorem. We omit the details.

[iii] Let $F$ and $G$ be defined by (4). Then $F, G$ share $(1,2)$.

Case-1. Suppose $H \not \equiv 0$. Then using Lemma 5 for $l=2$ in Lemma 4 we get

$$
\begin{gathered}
\left(\frac{n}{2}-1\right)[T(r, f)+T(r, g)] \leq 2[\bar{N}(r, 0 ; f)+\bar{N}(r, 0 ; g)]+ \\
+2[\bar{N}(r, \infty ; f)+\bar{N}(r, \infty ; g)]+S(r, f)+S(r, g) \leq \frac{4}{d}[T(r, f)+T(r, g)]+S(r, f)+S(r, g),
\end{gathered}
$$

which is a contradiction for $n>2+\frac{8}{d}$.

Case-2. Suppose $H \equiv 0$. This case can also be resolved same along the line of proof of Case-2 of Part [i] of this theorem.

Proof of Theorem 2. [i] Let $F$ and $G$ be defined by (4) with $f$ and $g$ as entire functions. Then we have $\bar{N}(r, \infty ; f)=S(r, f), \bar{N}(r, \infty ; g)=S(r, g)$ and $F, G$ share $(1,0)$. 
Case-1. Suppose $H \not \equiv 0$. Then proceeding in the same way like Case-1 of part [i] of the proof of Theorem 1 with $\bar{N}(r, \infty ; f)=S(r, f)$ and $\bar{N}(r, \infty ; g)=S(r, g)$ we obtain a contradiction for $n>2+\frac{7}{d}$. So we omit the proof.

Case-2. Suppose $H \equiv 0$. In this case, again using $\bar{N}(r, \infty ; f)=S(r, f), \bar{N}(r, \infty ; g)=$ $S(r, g)$ and Remark 3 if we proceed in the same way like Case-2 of part [i] of the proof of Theorem 1, then we obtain contradiction in all the subcases for $n>2+\frac{2}{d}$ except Subcase2.2.2. For Subcase-2.2.2 we make the following analysis.

For $d=1$, we get $2+\frac{4}{d}=6>4$. For $d=2$, we obtain $2+\frac{4}{d}=4>\frac{3}{2}+2$. For $d>2$, we always have $2+\frac{4}{d}>\frac{3}{2}+\frac{2}{d-1}$ as $\frac{3}{2}+\frac{4}{d}>\frac{3}{2}+\frac{2}{d-1}$. Hence in view of Lemma 7 and Remark 4 we obtain $f \equiv g$; i.e., $f_{1}^{d+r}=g_{1}^{d+s}$ for $n>2+\frac{4}{d}$. It is obvious that $2+\frac{7}{d}>2+\frac{4}{d}$. Hence this part is proved.

[ii] Let $F$ and $G$ be defined by (4) with $f$ and $g$ as entire functions. Then we have $\bar{N}(r, \infty ; f)=S(r, f), \bar{N}(r, \infty ; g)=S(r, g)$ and $F, G$ share $(1,1)$.

Case-1. Suppose $H \not \equiv 0$. Then proceeding in the same way like Case-1 of part[ii] of the proof of Theorem 1 with $\bar{N}(r, \infty ; f)=S(r, f)$ and $\bar{N}(r, \infty ; g)=S(r, g)$ we obtain a contradiction for $n>2+\frac{9}{2 d}$.

Case-2. Suppose $H \equiv 0$. Since $2+\frac{9}{2 d}>2+\frac{4}{d} ; \forall d \in \mathbb{N}$, so this case can be dealt same as in line of the proof of Case-2 of part-[i] of this theorem.

[iii] Let $F$ and $G$ be defined by (4) with $f$ and $g$ as entire functions. Then we have $\bar{N}(r, \infty ; f)=S(r, f), \bar{N}(r, \infty ; g)=S(r, g)$ and $F, G$ share $(1,2)$.

Case-1. Suppose $H \not \equiv 0$. Then proceeding in the same way like Case-1 of part[iii] of the proof of Theorem 1 with $\bar{N}(r, \infty ; f)=S(r, f)$ and $\bar{N}(r, \infty ; g)=S(r, g)$ we obtain a contradiction for $n>2+\frac{4}{d}$.

Case-2. Suppose $H \equiv 0$. Since $n>2+\frac{4}{d}$, so this case follows from the proof of Case-2 of part-[i] of this theorem.

5. Acknowledgement. The authors heartily thank the learned referee for his/her valuable suggestions towards the betterment of the paper.

\section{REFERENCES}

1. A. Banerjee, Uniqueness of meromorphic functions sharing two sets with finite weight II, Tamkang J. Math., 41 (2010), №4, 379-392.

2. A. Banerjee, Fujimoto's theorem - a further study, J. Contemp. Math. Anal., 51 (2016), №4, $199-207$.

3. A. Banerjee, I. Lahiri, A uniqueness polynomial generating a unique range set and vise versa, Comput. Methods Funct. Theory, 12 (2012), №2, 527-539.

4. A. Banerjee, S. Mallick, On the characterisations of a new class of strong uniqueness polynomials generating unique range sets, Comput. Methods Funct. Theory, 17 (2017), 19-45.

5. S. Bartels, Meromorphic functions sharing a set with 17 elements ignoring multiplicities, Complex Var. Elliptic Equ., 39 (1998), 85-92.

6. G. Frank, M. Reinders, A unique range set for meromorphic functions with 11 elements, Complex Var. Elliptic Equ., 37 (1998), №1, 185-193.

7. H. Fujimoto, On uniqueness of meromorphic functions sharing finite sets, Amer. J. Math., 122 (2000), 1175-1203. 
8. F. Gross, Factorization of meromorphic functions and some open problems, Proc. Conf. Univ. Kentucky, Leixngton, Kentucky (1976); Lecture Notes in Math., 599 (1977), 51-69, Springer (Berlin).

9. F. Gross, C.C. Yang, On preimage and range sets of meromorphic functions, Proc. Japan Acad. Ser. A Math. Sci., 58 (1982), №1, 17-20.

10. W.K. Hayman, Meromorphic functions, The Clarendon Press, Oxford, 1964.

11. H.H. Khoai, V.H. An, N.X. Lai, Strong uniqueness polynomials of degree 6 and unique range sets for powers of meromorphic functions, Internat. J. Math., 29 (2018), №5, 1850037-1-1850037-19.

12. I. Lahiri, Value distribution of certain differential polynomials, Int. J. Math. Math. Sci., 28 (2001), №2, 83-91.

13. I. Lahiri, Weighted sharing and uniqueness of meromorphic functions, Nagoya Math. J., 161 (2001), 193-206.

14. I. Lahiri, Weighted value sharing and uniqueness of meromorphic functions, Complex Var. Elliptic Equ., 46 (2001), 241-253.

15. S. Mallick, D. Sarkar, Unique range sets for powers of meromorphic functions, Mat. Stud., 50 (2018), №2, 143-157.

16. C.C. Yang, H.X. Yi, Uniqueness theory of meromorphic functions, Kluwer Academic Publishers, 2003.

17. H.X. Yi, A question of Gross and the uniqueness of entire functions, Nagoya Math. J., 138 (1995), 169-177.

18. H.X. Yi, Meromorphic functions that share one or two values II, Kodai Math. J., 22 (1999), $264-272$.

19. H.X. Yi, W.R. Lü, Meromorphic functions that share two sets II, Acta Math. Sci. Ser. B Engl. Ed., 24 (2004), №1, 83-90.

Department of Mathematics

Cooch Behar Panchanan Barma University

West Bengal, India

sanjay.mallick1986@gmail.com

smallick.ku@gmail.com 